\title{
La revocación en la Ley 39/2015, de 1 de octubre, de Procedimiento Administrativo Común de las Administraciones Públicas
}

\author{
Patricia Calvo López (España) \\ Universidad de Santiago de Compostela \\ patriciacalvo@avogacia.org
}

\section{NOTA BIOGRÁFICA}

Licenciada en Derecho con la especialidad en Derecho Público y Premio Extraordinario en el Máster de Derecho de las Administraciones e Instituciones Públicas, ambos títulos otorgados por la Universidad de Santiago de Compostela. Doctoranda en Derecho, Área de Derecho Administrativo, Universidad de Santiago de Compostela. Abogada en ejercicio.

\section{RESUMEN}

El objeto de la presente comunicación consiste en mostrar la situación actual en que se encuentra el instituto de la revocación, en el ámbito del procedimiento administrativo común, después de la entrada en vigor de la Ley 39/2015, de 1 de octubre, del Procedimiento Administrativo Común de las Administraciones Públicas. Para ello, se expondrá su ubicación sistemática dentro de la citada Ley, junto a la regulación del régimen de la invalidez, y se describirán las novedades, o ausencia de las mismas, contenida en la referida norma.

\section{PALABRAS CLAVE}

Invalidez, ineficacia, interés público, legalidad, oportunidad.

\begin{abstract}
The purpose of this text is to explain the current status of the revocation, within the scope of the common administrative procedure, after the Ley 39/2015, de 1 de octubre, del Procedimiento Administrativo Común de las Administraciones Públicas comes into force. For this, its systematic location will be exposed within the aforementioned Law, together with the regulation of the disability regime, and the novelties, or absence thereof, contained in said norm will be described.
\end{abstract}

\section{KEYWORDS}

Disability; ineffectiveness; public interest; legality; opportunity.

\section{SUMARIO}

I. INTRODUCCIÓN. II. LA REGULACIÓN DE LA REVOCACIÓN DENTRO DEL RÉGIMEN DE LA INVALIDEZ. III. LA REVOCACIÓN EN LA LPAC: AUSENCIA DE DESARROLLO. IV. CONCLUSIONES. V. REFERENCIAS BIBLIOGRÁFICAS. 


\section{INTRODUCCIÓN}

La revocación está presente en el Ordenamiento jurídico español desde hace largo tiempo, quizás la prueba más significativa de ello sea la previsión del artículo 16 del Reglamento de Servicios de las Corporaciones Locales de 1955 (desde ahora, RSCL), cuya vigencia se mantiene actualmente. Este precepto prevé específicamente la respuesta revocatoria, para el caso de las licencias que fueran otorgadas, cuando se produjera el incumplimiento de las condiciones a que estuvieran subordinadas, así como cuando se diera un cambio de circunstancias, por desaparición de las que motivaron el otorgamiento de la licencia o por el surgimiento posterior de otras que si hubieran existido al momento de dicho otorgamiento habrían justificado la denegación. Del mismo modo, el referido precepto ampara la aplicación del instituto revocatorio para los casos en que la Administración Local adopte nuevos criterios de apreciación, al tiempo que alude a la posibilidad de restituir «las cosas al ser y estado primitivo» cuando las licencias fueran otorgadas por error. Finalmente, el artículo 16 del RSCL reconoce el derecho al resarcimiento de los daños y perjuicios que se ocasionaran por la pérdida de efecto de las licencias para los casos del cambio de criterios de apreciación, así como en los casos de licencias otorgadas erróneamente.

Pocos años después del citado Reglamento se aprobó la Ley de procedimiento administrativo de 1958. Dicha Ley prometía a través de su exposición de motivos que «La Ley se ocupa por primera vez de un modo completo en nuestro ordenamiento jurídico de las potestades de la Administración respecto de sus propios actos, distinguiendo los supuestos de nulidad, anulación, revocación y rectificación de errores materiales y de hecho». No obstante, esta norma se limitó, en su artículo 112, a señalar que «Las facultades de anulación y revocación no podrán ser ejercitadas cuando por prescripción de acciones, por el tiempo transcurrido u otras circunstancias su ejercicio resultase contrario a la equidad, al derecho de los particulares o a las leyes». Es evidente que tal previsión dista mucho de constituir un régimen jurídico completo de la revocación.

La Ley 30/1992, de 26 de noviembre, de Régimen Jurídico de las Administraciones Públicas y del Procedimiento Administrativo Común (en adelante, LRJPAC), norma que sucede a la de 1958, también dedica a la institución revocatoria un único precepto, el artículo 105, sin contener, tampoco, la conceptuación de la revocación, ni exponer su régimen jurídico. La Ley 39/2015, de 1 de octubre, de Procedimiento Administrativo Común de las Administraciones Públicas (en lo sucesivo, LPAC), no varía esta situación, sino que reproduce, podría decirse que, sin cambios, el contenido del artículo 105 de la LRJPAC, que ahora encontramos en el artículo 109 de la LPAC. La única novedad consiste en una acotación temporal en el ejercicio de la acción revocatoria por parte de la Administración, antes abierta a "cualquier momento», y ahora, sujeta, aunque también lo estaba antes (ex artículo 106 LRJPAC), a los plazos de prescripción.

Habida cuenta de la carencia de una regulación acabada de la revocación, tiende a confundirse este instituto con otros conceptos cercanos, tales como el de la anulación, de los que conviene distinguirse, y dejar ya apuntada la diferencia esencial. Así, señala GONZÁLEZ PÉREZ (GONZÁLEZ PÉREZ, 1950: 150), citando a ROMANELLI, que mientras que «la anulación es la eliminación de un acto jurídico inválido del mundo del Derecho», «la revocación no supone un acto inválido, sino válido. Por ello, mientras la anulación encuentra siempre su fundamento en una causa extemporánea y consustancial a la emisión del acto, la revocación "encuentra su fundamento en causas sobrevenidas y objetivamente ciertas, que justifican la eliminación de un acto, válidamente nacido, de la vida jurídica.»

A los efectos de distinguir la anulación de la revocación, en el sentido apuntado por GONZÁLEZ PÉREZ, resulta ilustrativa la aclaración que efectúa CANO CAMPOS (CANO CAMPOS, 2017) sobre el concepto de validez y eficacia, de manera que distingue entre régimen de validez, en cuanto conjunto de requisitos necesarios para afirmar la validez de un acto; régimen de invalidación, como sistema de control o procedimiento para la declaración de la invalidez, y régimen de eficacia, referido a los efectos de la declaración de invalidez. Insiste CANO CAMPOS en que, un acto puede ser inválido, pero que, sin embargo, resulte inatacable su eficacia porque, por ejemplo, no se ha interpuesto el correspondiente recurso en plazo contra ese acto. En esos casos, señala el autor que, «no es que el acto haya devenido válido por el transcurso del tiempo, sino que no cabe la posibilidad de determinar administrativa o judicialmente si existe o no la causa de nulidad, por lo que quedarán consolidados los efectos que hayan derivado del acto.» (CANO CAMPOS, 2017: 7).

El supuesto de la revocación sería el contrario, un acto que nace válido pero que va a ser privado de eficacia.

Ante la insuficiencia de la regulación legal evidenciada, en cuanto al procedimiento administrativo común se refiere, pues existe regulación de la revocación en la normativa sectorial, resulta de interés, para aproximarse al concepto de la revocación, advertir cuáles son los motivos por los que cabría revocar un acto administrativo. 
Los motivos por los cuales un acto puede ser revocado pueden reconducirse a dos bloques, la revocación por motivos de legalidad y la revocación por motivos de oportunidad, siempre posteriores al nacimiento del acto administrativo válido. ALMEIDA CERREDA (ALMEIDA, 2010: 1.121 y ss.) describe los supuestos en que un acto inicialmente válido podría ser revocado, tratándose de un acto favorable para el interesado, al ponderarse junto al principio de irrevocabilidad de los actos favorables el principio de servicio objetivo a los intereses generales, que acoge el artículo 103.1 de la Constitución Española. En primer lugar, se hallarían «los supuestos en los que se incumplen por el administrado las condiciones inherentes al acto favorable dictado ${ }^{1}$ », puntualizando que el término "condición» ha de entenderse en el sentido de "situación o circunstancia indispensable para la existencia de otra, y no con el significado de acontecimiento futuro e incierto del que por determinación legal o convencional depende la eficacia inicial o la resolución posterior de ciertos actos jurídicos» ${ }^{2}$ En segundo lugar, un acto sería revocable cuando «se produce una modificación de las circunstancias en atención a las cuales se ha dictado el acto». Finalmente, el citado autor alude a los casos en que la Administración, «en aras a prestar un mejor servicio público, decide cambiar los criterios conforme a los cuales se ha dictado el inicial acto favorable».

Igualmente, interesa apuntar otros supuestos en que se puede producir la revocación de actos favorables a los interesados, tales como, siguiendo a ALMEIDA CERREDA (ALMEIDA, 2010: 1.130), los supuestos en que al propio particular interese la revocación de un acto, porque, por ejemplo, ya no obtiene provecho del acto y su mantenimiento le produce un coste. En este caso, se impone el principio de disponibilidad de los derechos sobre el principio de irrevocabilidad de actos favorables, debiendo entender que, naturalmente, al interés público conviene, o no le es perjudicial, dejar sin efectos el acto. Y, para terminar, se encuentran entre los supuestos de revocación, los casos legalmente previstos como sanción, en que ante la concurrencia de determinada circunstancia se impone la consecuencia sancionadora de la revocación del acto, que no deben ser confundidos con la revocación por incumplimiento de las condiciones impuestas, como recuerda ALMEIDA con cita de la Sentencia del Tribunal Supremo de 28 de septiembre de 2005, donde se destacan las características del derecho administrativo sancionador frente a la revocación por incumplimiento de condiciones, tales como sujeción a los principios de legalidad y tipicidad, procedimiento especial -sancionador(antes, en la Ley 30/1992, especialidad del procedimiento común, ahora, con la Ley 39/2015, y junto a la responsabilidad patrimonial, procedimiento especial), principio de responsabilidad y culpabilidad, y plazos específicos de prescripción.

Sin perjuicio de reconocer la existencia de la revocación de actos favorables en el Ordenamiento jurídico español, lo cierto es que el artículo 109.1 de la LPAC sólo se refiere, al igual que lo hacía el artículo 105 de la LRJPAC, a los actos de gravamen o desfavorables para los interesados, lo que, por parte de la doctrina, ha sido visto como la consagración normativa del principio de irrevocabilidad de los actos favorables (ALMEIDA, 2010: 1.111). A pesar de la literalidad del artículo 105 de la LRJPAC, en el Ordenamiento jurídico español existen numerosas demostraciones, dentro de la legislación sectorial, de la revocabilidad de actos favorables (vid. ALMEIDA, 2010: 1.123).

Además de circunscribir la revocación a los actos desfavorables, el artículo 109, al igual que su predecesor, 105, impone como requisitos a la revocación que ésta no constituya dispensa o excepción no permitida por las leyes, ni sea contraria al principio de igualdad, al interés público o al Ordenamiento jurídico. Imponiendo, finalmente, y agotando la regulación legal existente de la revocación en el ámbito del procedimiento administrativo común, un límite temporal a la revocación, «que no haya trascurrido el plazo de prescripción». Cabe destacar que este límite temporal representa el único cambio del régimen normativo común de la revocación observado tras la derogación de la LRJPAC y la entrada en vigor de la LPAC, pues el artículo 105 de aquélla permitía a las Administraciones Públicas «revocar en cualquier momento», a salvo los límites del artículo 106 (prescripción de acciones, transcurso del tiempo u otras circunstancias por las que su ejercicio resultara contrario a la equidad, a la buena fe, al derecho de los particulares o a las leyes) que se entendían aplicables a la facultad revocatoria y que hoy reproduce el artículo 110, por lo que no se puede entender que exista un verdadero cambio en la regulación, sino que el límite de la prescripción ya se entendía aplicable, se dijera o no de forma directa en el propio precepto que se refiere expresamente a la revocación.

1 IGLESIAS GONZÁLEZ (IGLESIAS, 2017: 78 y ss.) se refiere también a los supuestos de incumplimiento sobrevendido de los requisitos exigidos, como supuestos susceptibles de ser conceptuados bajo el paraguas de la revocación, se trata de actos cuyos requisitos se atendieron inicialmente, pero dejaron de cumplirse después de nacido el acto, por lo que éste puede revocarse.

2 En este mismo sentido, IGLESIAS GONZÁLEZ (IGLESIAS, 2017: 86 y ss.) sustituye el término «condición» por el de «obligación» impuesta al interesado. 


\section{LA REGULACIÓN DE LA REVOCACIÓN DENTRO DEL RÉGIMEN DE LA INVALIDEZ}

La nueva Ley reguladora del Procedimiento Administrativo Común de las Administraciones Públicas, la Ley 39/2015, de 1 de octubre, acoge, al igual que su predecesora, la Ley 30/1992, de 26 de noviembre de Régimen Jurídico de las Administraciones Públicas y del Procedimiento Administrativo Común, el único precepto que se refiere a la revocación de actos (artículo 109) dentro del Capítulo I, del Título V, esto es, contemplándose dentro del régimen de la revisión de oficio de los actos administrativos.

Inicialmente, podría pensarse que la ubicación del precepto que la LPAC dedica al instituto de la revocación no se encuentra justificado, por cuanto la revocación presupone la existencia de un acto válido en origen, como se ha señalado anteriormente, y por tanto, distanciado de los supuestos de nulidad y anulabilidad a que se refiere el Capítulo I, del Título V. No obstante, hay que destacar que perfeccionada la revocación, el acto originalmente válido devendrá ineficaz, y esta importante consecuencia, la de la ineficacia del acto administrativo, que determina que deje de producir efectos, es la que acerca el instituto revocatorio a los supuestos en los que se priva de eficacia al acto, luego de examinar los requisitos de validez en origen (nulidad y anulabilidad).

Asimismo, si acudimos a los códigos de procedimiento de los Estados de nuestro entorno jurídico, señaladamente, Portugal, Italia y Francia, observamos como, al igual que en España, el instituto revocatorio se regula junto al régimen de la invalidez o seguidamente a él. Así, en el Decreto/ Ley 4/2015, por el que se aprueba el Código del Procedimiento Administrativo de Portugal, se regula la revocación, dedicándole la Sección IV, «Da revogaçao do acto administrativo», luego de exponer el régimen de la invalidez (Sección III), dentro del Capítulo II, «Do acto administrativo», que se inscribe en la Parte IV del Código, «Da actividade administrativa». Igualmente, junto a la regulación de la revocación, a la que el Código portugués destina diez artículos (138-147), la Sección IV se cierra con el artículo 148, dedicado a la rectificación de errores, guardando así paralelismo con la LPAC, que en el apartado 2 de su artículo 109 se refiere, precisamente, a la rectificación de los errores materiales o de hecho, que sin embargo no comparten naturaleza jurídica con la revocación. En el caso de Italia, la Ley 241/1990, que aprueba la «Nuove norme in materia di procedimento amministrativo e di diritto di accesso ai documenti amministrativi», con las modificaciones operadas por el artículo 14 de la Ley 15/2005, introduce en la Ley 241/1990 el Capítulo IV bis (artículos 21 bis a 21 nonies), rubricado "Efficacia ed invalidità del provvedimento amministrativo. Revoca e recesso.», cuyo gráfico título evidencia la regulación conjunta que se viene afirmando. Por su parte, el «Code des relations entre le public et l'a administration», de 2015, sin recoger la revocación expresamente, atiende en su Título IV a «La Sortie de vigueur des actes aministratifs», refiriéndose a la abrogation (modificación o desaparición en el futuro del acto administrativo) y al retrait (modificación o desaparición retroactiva de una decisión administrativa) de forma vinculada.

\section{LA REVOCACIÓN EN LA LPAC: AUSENCIA DE DESARROLLO}

A la hora de abordar las novedades introducidas por la LPAC en materia de revocación, cabe referirse más bien a la inexistencia de novedades respecto de la regulación contenida en la LRJPAC. Como ya se ha advertido, el único cambio que presenta el artículo 109 de la LPAC respecto del artículo 105 de la LRJPAC reside en que éste otorgaba la facultad de revocar el acto en cualquier momento, mientras que aquél introduce la limitación temporal derivada de la prescripción, en cuanto garantía de seguridad jurídica, si bien, ya se entendía entonces de aplicación por determinación del artículo 106 de la LRJPAC, cuyo contenido se reproduce ahora en el artículo 110 de la LPAC, que igualmente se dice de aplicación a las «facultades de revisión establecidas en este Capítulo».

La referida situación ha merecido la crítica de la doctrina que no deja de apuntar a la oportunidad perdida por el legislador para acabar con la ambigüedad que persigue a esta figura a la hora de interpretar su alcance y fundamento, además de la falta de sistematización y clarificación conceptual, como señalan ALENZA GARCÍA (ALENZA, 2017: 183) e IGLESIAS GONZÁLEZ (IGLESIAS, 2017: 61).

Así, la regulación de la revocación en el ámbito del procedimiento administrativo común se residencia en la ausencia de regulación completa y acabada, lo que plantea serios problemas a la hora de articular y aplicar el instituto revocatorio. Como enseña ALENZA (ALENZA, 2017: 188), en primer lugar, la LPAC no determina cuál es el órgano competente para la revocación. Asimismo, se critica que la acción revocatoria se circunscriba a la actividad de oficio de la Administración y no se prevea la revocación a solicitud de los 
interesados, abriendo la posibilidad de su ejercicio por vía del derecho de petición (ALENZA, 2017: 189), aunque esta vía observará limitaciones frente a los supuestos en que se encuentra expresamente previsto la actuación de los particulares con el efecto de iniciar un procedimiento, en este caso, el que conduzca a la declaración de la ineficacia del acto originariamente válido. Como ya se ha advertido, y señala el citado autor, en la previsión del artículo 109 de la LPAC se dejan al margen de la revocación los actos favorables a los interesados. Cierto que no existe un procedimiento estandarizado para los supuestos de revocación, aunque se salvaría el defecto de regulación específica por medio de la aplicación del procedimiento administrativo común -iniciación, instrucción, finalización-, (ALENZA, 2017: 204). Finalmente, queda sin resolver si la revocación del acto tendrá efecto retroactivo o no y la posibilidad de recurrir la resolución que acuerde la revocación a lo que tampoco se da respuesta concreta (ALENZA, 2017: 208), así como la relación de la revocación con la responsabilidad patrimonial, que sí está prevista para la anulación de actos.

\section{CONCLUSIONES}

Como conclusiones a esta comunicación sobre la situación de la regulación de la revocación en la Ley 39/2015, de 1 de octubre, del Procedimiento Administrativo Común, cabe destacar, en primer lugar, la insatisfactoria respuesta a la doctrina que tiene reiteradamente mostrado la necesidad de completar el régimen jurídico de la revocación dentro del procedimiento administrativo común, acabando con la ambigüedad que reinaba hasta el advenimiento de la Ley 39/2015 y que, sin embargo, se mantiene sin cambios.

En segundo lugar, afirmar la correcta ubicación de la revocación, dentro de la regulación del régimen de la invalidez, tal y como respalda el derecho comparado, aunque con ciertas matizaciones en atención a algún problema concreto que dicha situación pueda plantear.

\section{REFERENCIAS BIBLIOGRÁFICAS}

ALENZA GARCÍA, J. F. (2017): Revisión, revocación y rectificación de actos administrativos, Pamplona: Aranzadi, S.A.U.

ALMEIDA CERREDA, M. (2010): "El principio de irrevocabilidad de los actos favorables", en SANTAMARÍA PASTOR, J. A. (dir.), Los principios jurídicos del Derecho administrativo, Madrid: La Ley.

CANO CAMPOS, T. (2017): “El laberinto de la invalidez: algunas pistas para no perderse", en Indret, núm. 4, págs. $1-53$.

GONZÁLEZ PÉREZ, J. (1950): “La revocación de los actos administrativos en la jurisprudencia española”, en RAP, núm. 1 , págs. $149-161$.

IGLESIAS GONZÁLEZ, F. (2017): La revocación de actos administrativos favorables, Valencia: Tirant lo Blanch. 